\title{
Unmet medical care needs in persons with multiple chemical sensitivity: A grounded theory of contested illness
}

\author{
Pamela Reed Gibson * Britney Leaf, Victoria Komisarcik \\ Department of Psychology, James Madison University, Harrisonburg, VA, United States
}

Received: October 20, 2015

Accepted: December 15, 2015 Online Published: January 12, 2016

DOI: $10.5430 /$ jnep.v6n5p75

URL: http://dx.doi.org/10.5430/jnep.v6n5p75

\begin{abstract}
We interviewed nineteen persons with self-reported multiple chemical sensitivities in order to explore medical care access and use for this population with a "contested" illness. Interviews were analyzed using grounded theory in the tradition of Glaser, via incident, focused, and theoretical coding. We named the core category health quest (resisting annihilation) and construed four categories that embody the essence of this quest: illness as omnipotent, bidding for access, standard protocols irrelevant, and transcending hegemony. The categories suggest that mainstream medicine is unprepared to care for this population, that standard interventions are ineffective but used regardless, and that persons feel that they must survive on their own outside of the structured medical care system. In fact, most informants avoided medical care unless faced with an emergency. Results are discussed in terms of the need to recognize and understand this health problem.
\end{abstract}

Key Words: Multiple chemical sensitivity, Chemical intolerance, Chemical hypersensitivity, Environmental sensitivity, Contested illness

\section{INTRODUCTION}

The nature of contested illness is such that it has not yet fully entered the official diagnostic code and hegemonic thinking of mainstream medical care. Hence patients are not uniformly seen as having legitimate physical illness and they face disbelief and psychological attributions from others while the mainstream medical system debates the validity of the condition. Dumit explored the context of institutional codes and patient-doctor interactions and how they contribute to the legitimacy, or lack thereof, for illnesses not yet institutionalized in culture, and characterized these conditions as "Illnesses you have to fight to get."[1] Multiple Chemical Sensitivity (MCS) is one such condition/contested illness that is also referred to as chemical intolerance (CI), environmental sensitivity (ES), chemical sensitivity (CS), and chemical hypersensitivity, among other names. MCS is a chronic, multi-sensory illness/disability caused by negative reactions to chemicals - even those in ambient air. ${ }^{[2,3]}$ The most commonly used case definition of MCS has six criteria: low levels of exposure to chemicals well under toxicity levels that produce symptoms, symptoms are reproducible with repeated exposure to the chemical, the condition is chronic, symptoms lessen or improve when the chemicals or triggers are removed, similar symptoms might be caused by several chemically unrelated substances, and symptoms involve multiple organ systems. ${ }^{[4]}$ Persons report developing MCS from

\footnotetext{
*Correspondence: Pamela Reed Gibson; Email: gibsonpr@jmu.edu; Address: Department of Psychology, James Madison University, Harrisonburg,
} VA, United States. 
one large chemical exposure, ongoing low-level exposures, physical illnesses, and combinations of factors. ${ }^{[3]}$

People with MCS typically experience a spreading and broadening of symptoms and sensitivities, in which they become intolerant to an increasing number of incitants, first those related to the initial sensitizing exposure, later to those unrelated. ${ }^{[2]}$ Gibson and Vogel found the most common incitants to be pesticide, formaldehyde, fresh paint, new carpet, diesel, exhaust, perfume, and air fresheners, and the most prevalent symptoms to be tiredness/lethargy, difficulty concentrating, and long-term fatigue. Other symptoms reported by these authors included muscle aches, digestive problems, joint pain, headache, irritability, tenseness, feeling "spacey", sleep problems, depressed feelings, and many others. ${ }^{[5]} \mathrm{A}$ portion of persons with MCS also report having electrical hypersensitivity (EHS), also poorly understood and having serious life impacts. Persons with EHS must avoid electromagnetic frequencies such as computer screens, florescent lighting, microwaves, wireless technology, and in extreme cases, sometimes any use of electricity.

Despite its contested nature in industrial cultures that value technological products, MCS has been identified and studied internationally. In a Swedish community study, 33\% reported odour intolerance and 19\% had experienced "affective and behavioral consequences". [6] Also in Sweden, Andersson and colleagues found that of 402 teenagers, $15.6 \%$ reported having chemical intolerance and $3.7 \%$ reported having life consequences of the condition. ${ }^{[7]}$ Berg and others found a higher prevalence among 1,911 Danes, where 27\% reported multiple symptoms from everyday chemical exposures and $3.3 \%$ reported having made life changes. ${ }^{[8]}$ In Germany, $32 \%$ of persons reported chemicals as causing health complaints and $9 \%$ endorsed that their body "reacted immediately". [9] The first US household population study found that $33 \%$ of adults reported some chemical intolerance and $3.9 \%$ reported becoming ill every day from exposures. ${ }^{[10]}$ In 2003 , $12.6 \%$ of persons in a US household population study reported chemical sensitivity. ${ }^{[11]}$ Alarmingly, Katerndahl and others surveyed 400 persons in medical waiting rooms in the US using Miller's Quick Environmental Exposure and Sensitivity Index (QEESI) and 20.3\% met the criteria for chemical intolerance. ${ }^{[12]}$ Cui and colleagues (2014) found much lower prevalence rates in two Japanese factories $(1.1 \%$ and $2.4 \%$ ) using Miller's criteria, but the authors believe that the "healthy worker effect" would extrude from the workplace most of those who become ill from exposures. ${ }^{[13]}$

Despite the growing number of prevalence and descriptive studies of MCS, there is no agreement regarding etiology. Dantoft and others (2015) have recently reviewed the hy- potheses and research regarding causes, including neurogenic inflammation, neural sensitization, immune dysfunction, olfactory cuing, genetics, and even psychogenic theories. ${ }^{[14]}$ For example, to cite just one theory, upper airway inflammation has been found in some studies of persons with MCS, but not in others. ${ }^{[15,16]}$ A full discussion of etiology is beyond the scope of this paper and is complicated by many factors. However, a promising strategy for study seems to be real-time monitoring of volatile organic compound (VOC) exposure and its correlation with symptoms. ${ }^{[17]}$ It does seem to be a somewhat stable finding that CS overlaps strongly with allergy and asthma. ${ }^{[18,19]}$

People who are severely disabled with MCS experience drastic life change as a result of their inability to tolerate common chemical exposures that have come to be ubiquitous in workplaces, medical facilities, and community settings. Many people are unable to work, suffer financial devastation, and have trouble finding housing. Individuals with MCS cope with the illness by striving to create a safe environment, free of chemicals and triggers. However, public environments are rarely chemical-free. As a result, people might be limited regarding work, ${ }^{[20,21]}$ public access, ${ }^{[22]}$ and social interaction, ${ }^{[23,24]}$ and may experience considerable personal distress. Fox and Kim (2004) summarized the plight of those with MCS: "The barriers faced by individuals from emerging disability groups often prevent experiencing the benefits of participation in society" (p. 325). ${ }^{[25]}$ Thus quality of life has been found to be low, even when compared with groups who experience other chronic illnesses. ${ }^{[5,26]}$

In addition, people with MCS encounter many barriers when attempting to access medical care. Patients who are chemically intolerant reported seeing an average of 8.6 practitioners each, but perceived only a quarter of them as helpful. ${ }^{[27]}$ MCS does not fit in the dominant biomedical paradigm, as it typically manifests as an invisible disability. Moreover, there is a lack of knowledge about MCS among practitioners and the general public. It is problematic that no etiology, specified treatment, or official diagnosis of MCS is recognized in mainstream medicine. ${ }^{[28]}$ Thus, people with MCS have difficulty accessing adequate care and many of their medical needs remain unmet. In addition, those who also experience EHS are often completely unable to enter the high-tech world of current medical care due to electromagnetic exposures from scanners, computers, and other medical equipment. ${ }^{[29]}$

Qualitative studies of contested illness have shed light on the personal struggles of persons whose bodies defy accepted categories. Clark and James found that participants with Chronic Fatigue Syndrome (CFS) suffered personal loss in 
their struggle to find medical care, as they lost sight of their sense of self, and their relationships with others were drastically affected. ${ }^{[30]}$ Similarly, Crooks discussed the shrinking "lifeworlds" for women with fibromyalgia, ${ }^{[31]}$ and Gibson, and others found striking identity changes in persons with MCS. ${ }^{[32]}$

In a grounded theory analysis, Swoboda found that "rejection, investigation, confirmation, and explanation" were all overarching themes in the lives of participants managing a contested illness. In addition to these categories within contested illness, Swoboda found a shared conceptualization of contested illness among the participants. This conceptualization included experiencing a(n) "1) array of symptoms, 2) varying course, 3 ) the lack of an accepted testing measure to diagnose it, and 4) that the illness was 'reflective of human vulnerability to dangers increasingly encountered in modern life'"'(p. 244). ${ }^{[33]}$

In the current study, we were interested in the experience of acquiring and managing medical care among persons with MCS. It is important to study MCS, as the research to date demonstrates considerable suffering, disability, and lack of access to crucial resources for this group. Medical care, in particular, is vital, and yet reportedly unavailable for persons who cannot tolerate common chemicals in the medical setting. ${ }^{[20,26,28]}$ We followed the methods of grounded theory as presented by Barney Glaser. ${ }^{[34,35]}$ In grounded theory, researchers avoid projecting any predetermined ideas and/or formulating a hypothesis prior to conducting research. Rather, grounded theory allows prominent themes to emerge from the data, which make up the foundation of the overall theory. The process of grounded theory consists of generating theory from the data and then being guided by that emerging theory in continuing the research. The researcher must act as an analyst when collecting and coding data, generating memos, and integrating categories and properties into the data. Categories emerge which fit the nature of the data and are illustrated by properties, which are relevant to the categories. Grounded theory attempts to highlight the problems and processes of a certain topic to interpret and understand the data. The core category is seen as that which occupies the major efforts of the informants.

We chose Glasser's GT because we hypothesized that persons with MCS would share processes with persons with other contested illnesses. We therefore sought to discover broad theory that would apply to the process of attempting to acquire medical care for MCS, which we conceptualized as a contested illness in the United States-that is, an illness that has not achieved complete legitimacy in the medical arena.

As a researcher of contested illness, author one is familiar with MCS and with the MCS community. Though this lab (author one) has studied MCS for many years,, and though this is more easily said than done, we attempted to set aside any preconceived notions of what the central process and categories might be. To do this we gave equal weight to the perceptions of authors two and three, and stuck close to the data provided by the interviews. After concluding the study, author one felt that, despite listening to persons with MCS for many years, the exact terms of the core categories were not phrases that she would have articulated prior to this study. In fact no research participant has used the exact phrase that emerged as the theoretical aspect of our core.

\section{METHOD}

\subsection{Participants}

As part of a larger survey study of unmet medical needs in $\mathrm{MCS}^{[28]}$ conducted on Qualtrics, an online survey maker, participants were asked to indicate whether they were willing to be contacted for a follow-up, in-depth, interview study of unmet medical needs. We chose telephone interviews because of the large geographical distribution of the participants in the large study and because of the difficulty of traveling and accessing buildings/settings for those with MCS. Of the 82 participants who volunteered to be contacted, we were able to interview 19 persons (14 women and 5 men) who selfidentified with chemical intolerance. The mean age of participants was 50.7 years; persons had been chemically sensitive for a mean of 19 years. Six participants had graduate level degrees, seven had four-year college degrees, four had completed some college coursework, and one had a high school diploma. Fifteen participants identified as straight, two as gay, and two identified as bisexual. Participants were primarily unemployed (14), with two participants working part-time from home, one working part-time outside the home, one working full-time outside the home, and one working fulltime from home. Eight were single, four were living with a partner, three were divorced or separated, three were married, and one was widowed.

\subsection{Procedure}

After receiving permission from our Institutional Review Board, we contacted all of the 82 persons who had agreed to be interivewed. Nineteen persons responded, agreeing to proceed, and were scheduled via email for telephone interviews. Participants were phoned at the appointed times, verbal permission was elicited for the interview, and taped interviews lasting 30-45 minutes were conducted. Interviews were transcribed, and coded via incident, focused, and theoretical coding. The process was iterative, as the three researchers met weekly, discussed our coding, refined our questions, conducted further interviews, generated memos, and refined our 
codes. We relied heavily on visual diagrams to represent our core, its categories, and properties. Additionally we left the review of recent literature on contested illness until the end. Through the constant comparisons method, categories and their properties emerged until we reached a "stable" plateau where they were saturated and no new instances of their properties emerged. We did not force data nor approach the project with any hypothetical categories, but instead allowed them to emerge. The last few interviews were theoretically sampled to validate or interrogate our categories-that is, we explored our categories in persons with somewhat different circumstances, attempting to conceive the boundaries of our categories and any conditions that influenced them. Though theoretical sampling did not alter our model, we recognize, as with all qualitative modeling, that this in only one construction of how the process of medical care for persons with contested illness might look.

\section{Results}

We named our core variable (that which drives behavior for participants) Health Quest, the behavioral concomitant of attempting to Resist Annihilation (theoretical). The four categories that embodied the attempt to survive a contested and poorly integrated illness were: Illness as Omnipotent (Threatened Annihilation), Bidding for Access (The Quest for Validation), Standard Protocols Irrelevant (Lacking a Medical Paradigm), and Transcending Hegemony (Avoiding Annihilation).

\subsection{Illness as omnipotent}

Category one, illness as omnipotent, had three properties: realization, struggling with drastic change, and desperation. Persons who experience MCS as a contested illness face threatened annihilation (theoretical) as they realize the gravity of their situation. Realization is associated with having frequent symptoms and life-threatening reactions, and even seeing others die from illness-related reactions. The growing intrusion of the condition and seeing its effects in others substantiate the threat; persons come to know that they are facing a very rough life and experience fear, vigilance, and restriction of personal potential.

The struggle with drastic change embodies diminishing access, dwindling resources, often-worsening symptoms, and the experience of physical pain. As one participant described, "I went from super athletic in college, played basketball in college, to [highly skilled occupation] to uncoordinated and having to give up my license all because of some condition that they don't even acknowledge exists." The combination of vigilance to avoid exposure and experiencing intrusive symptoms leads to isolation, which enhances the struggle with drastic change and can lead to the property of desperation. The isolation, threat and struggle are illustrated in the statement of one informant: "everything has the potential to ruin your day".

\subsection{Bidding for access}

In category 2 , bidding for access, the attempt is made to access health care. In the quest for validation (theoretical) the person approaches the conventional medical system to validate and diagnose an illness. Bidding for access has four properties: seeking providers, preparing for the appointment, lacking resources, and exclusion/irrelevance. Providers are phoned and questioned about chemical barriers in the office and to request accommodations for the appointment. Sometimes it is clear from the call that the office will not be accessible. At times applicants are mislead regarding the presence of barriers. Even if an office seems accessible, persons often experience anxiety, attempt to prepare mentally for the appointment, and often have to schedule "downtime" to recover afterward. The anxiety relates to how one will attend an appointment without enduring a disabling reaction. Preparing mentally might include worrying, making notes about symptoms so that one can communicate even if a neurological reaction ensues, and perhaps preparing a mask to wear to the appointment.

In the case that no accessible facilities can be found, persons are excluded from the system before even attending an appointment. For those who acquire an appointment, the experience might involve dismissal and belittlement throughout. As one informant recounted

They're very dismissive and you hear them talk about you in the hallway, you see them rolling their eyes, oh yeah the woman in there with all the chemical things. All we're trying to do is you know, keep breathing. I actually had an anaphylactic reaction in front of a doctor and he told me that it was in my head.

Likewise, those who lack resources, such as health insurance, might face exclusion regardless of the accessibility of the facilities. Facing the impossibility of even getting into the system, some are made irrelevant before even attending a medical appointment.

\subsection{Standard protocols irrelevant}

Category 3, standard protocols irrelevant (lacking a medical paradigm), has 4 properties: unpredictable reactions, misdiagnosis, extrusion, and avoiding medical care. Western industrial culture lacks a medical paradigm for the risks of chemical exposure in general and for the risks of low-level 
exposure in particular. Therefore, when bidding for access, persons are often met with random and unpredictable reactions from health care providers, including blame for their illness or disbelief, which push the patient out of the medical care system (extrusion). At times they receive support, which allows them to keep pursuing medical care. But all too often they meet with misdiagnosis, and are perceived as having psychiatric conditions. The patient, a non-responder to the available "toolbox", is delegitimized and misdiagnosed-largely perceived as psychiatric. “... like I was crazy, or they want to attach some type of psychological label to it. Or they don't really hear what I'm saying, so they'll prescribe a prescription when they know I can't take it."

In the absence of relevant treatments, existing treatments/protocols might be forced on the patient, regardless of their safety or relevance to the patient's sensitivities. Lacking an understanding of the condition, providers offer standard approaches that embody risks for persons unable to tolerate medications, anesthetics, and other approaches. For example, one person experienced anaphylaxis from epinephrine and was pronounced dead by attending personnel, yet later found to be alive by the nurse who came to "prepare the body".

Even attending the appointment leaves many with symptoms from chemical exposures in the office, and, for some, even interferes with their communication with the provider:

So for me that's kind of a typical thing I go somewhere and feel fine before I go in then I get there and just don't even have a very good discussion with the physician because I just feel kind of spacey when I leave.

Iatrogenic harm from exposure or treatment then leads to avoiding medical care, with its attendant negative consequences. All respondents stated that they avoided conventional medical care unless they had an emergency.

\subsection{Transcending hegemony}

Hegemony is defined by Purdue University as "The process by which dominant culture maintains its dominant position: for example, the use of institutions to formalize power; the employment of a bureaucracy to make power seem abstract (and, therefore, not attached to any one individual): the inculcation of the populace in the ideals of the hegemonic group through education, advertising, publication, and so forth; the mobilization of a police force as well as military personnel to subdue opposition." ${ }^{\text {[36] }}$

Patients ultimately move toward self-sufficiency and thus transcend hegemony through self-education, self-advocacy, and finally, self-empowerment. Persons access online resources, learn to clean up their home environments, avoid

Published by Sciedu Press chemical exposures, access conventional care only in emergency, and adopt a paradigm of MCS as an environmental health issue. The variety of negative reactions received in category 3 teaches that disclosure must be managed as it becomes evident that disclosure of chemical sensitivity destroys credibility (theoretical). Disclosure is managed through telling only accepting providers, reporting only necessary symptoms to questionable providers, and thus "playing the game". Conditions were evident in informants' descriptions of how they managed their disclosure. The mention of having MCS would often cast a negative light on the provider's perception of the patient; thus a "good" patient does not bring up "contested" conditions or mention conditions outside the provider's purview.

I do not use the term MCS and I don't even feel required to give any kind of diagnosis. I generally talk about it in terms of limitations in accordance with the ADA, in order to obtain some level of understanding...

Through managing disclosure in this manner one can acquire limited help without delegitimization.

If the patient finds a sympathetic provider, there is more leeway, and disclosure need not be so restricted. Even if this is the case, however, and symptoms are treated, the patient often has to endure symptoms from chemical barriers in the facilities.

Positioning the condition as an environmental issue means adopting a worldview situating the person not as a delegitimized and extruded patient, but as someone for whom the medical system is not yet ready. In becoming self-sufficient, persons become self-empowered and move away from selfdoubt that resulted from a lack of medical legitimacy. One participant described engaging in self-advocacy: "I've had to learn how to be more assertive and to say, 'these are my requirements, this is my health." Another participant described adopting a more accepting worldview: "I eventually grew to trust myself and my experiences. Like look, there's something wrong and it's not in your head and that's it. I eventually grew to trust myself."

Persons also sought out alternative providers, and in this way, some who were fortunate got substantial help. One respondent described moving from self-doubt after finding an accepting provider:

I think my biggest problem was that I continued to question myself. And his response was "who wouldn't? You are given a rotten diagnosis for which there is no cure and no real solution, you feel like hell all the time, of course you want it 
to be something that can be solved." I thought oh boy did I ever pick the right person.

Otherwise, medical care is avoided except in the case of emergency. Through chemical avoidance, and limited, or substantial alternative help, the person survives largely outside of the conventional medical system. This strategy can be largely stable for long periods. However, the temporal nature of our categories is exposed by the fact that should an emergency occur, it is necessary once again to bid for access and face irrelevant protocols.

\section{DiscuSSION AND CONCLUSION}

All people confront annihilation daily through risks, struggles, even threats of which they are unaware. All chronic and acute illnesses would seem to present more conscious struggles. Contested illnesses, situated outside the medical paradigm, present patients with yet additional challenges. With MCS, the life-threatening nature of reactions and the inability to access useful medical care, increase the intensity of the annihilation threat. Thus, though health quest is a common process relevant to any illness or challenge, the underlying theoretical core resisting annihilation gives this contested illness an intensity and urgency not seen in chronic illness that is better understood and treated. The threat permeates all categories and weaves them together in a process of separation from the conventional medical community as much as possible as a result of extrusion and becoming self-sufficient. Thus patients go from realizing that their condition is ominous to attempting to enter the medical system to struggling as a contested patient to eventual attempted self-sufficiency.

Glasser said, "Society is a cumulative build up of normative models for thought, feeling and behavior. This build up forces routine preconceptions by people as they act and go through life" (p. 83). ${ }^{[34]}$ Therefore, "The social structure of the way things are will always win and anybody who tampers with it will be taken care of usually by subtle forms of ostracism" (p. 89). Persons with MCS present a "reality" outside of the medical-social structure and are often dealt with accordingly. The established medical care system trains people through a series of seemingly random, negative inputs, not to depend on it, thwarting any expectation of "getting well". It silences people about their MCS except in "special company" where it is safe to disclose. People with MCS might initially believe the "properline", ${ }^{[34] ~ " I f ~ I ~ f i n d ~ t h e ~}$ right practitioner I will get well." But this breaks down over time through belittlement and ostracism. Finally "getting it", people depart, possibly seeking alternatives, becoming self-reliant, and subtracting themselves from the system be- cause they experience MCS, a disconfirming piece of data that fails to fit into medical hegemony. Svoboda ${ }^{[33]}$ similarly found a core category of "illness legitimacy" in persons with CFS, MCS, or GWS who described their initial treatment as inadequate and who later pursued other avenues.

The attempts of medical providers to work with patients with irrelevant protocols and categories echoes Dummit, "Squeezing a variable illness into exceptionally small bureaucratic holes requires conforming one's variability to the strict requirements of the micro-judgments of administrative experts" (p. 585). ${ }^{[1]}$ And consequences for patients were overwhelmingly negative, including drug reactions, and in one case (described earlier) apparent death until medical personal realized that she was indeed alive. Fox and Kim critique conventional services as such,

As so-called "emerging disability populations" knock on the door of service providers whose systems are designed to work with "traditional disability populations", greater efforts must be made to more clearly understand what emerging disabilities are in order for these service delivery systems to remain relevant (p. 324). ${ }^{[25]}$

But those efforts seem yet to be made. Gibson and Lindberg found that though $97 \%$ of 90 physicians had encountered a patient with chemical intolerance, only $7 \%$ were satisfied with their knowledge of the illness, and only $6 \%$ had any treatment protocol to offer these patients. ${ }^{[37]}$

Understandably, disclosure is a conundrum for those with contested illness, and congruent with Joachim and Acorn's discussion of having to choose between passing as normal or facing stigma, our participants managed disclosure closely to avoid the consternation of conventional providers. ${ }^{[38]}$

Jussila found the core categories could be a progression or could vary and be four separate behavior patterns according to situational variables. ${ }^{[39]}$ Our categories also seem to progress, but not necessarily in all conditions. For example, one could get stuck in "bidding for access", asking repeatedly for help from marginalizing providers. Despite the lack of forthcoming support, some continue to try. Others become intensely angry at the lack of a medical paradigm and make it their goal to educate doctors. Some move more quickly to transcending hegemony and become self-sufficient. Conversely, if one succeeds in finding care, there is less need to become self-sufficient.

Glaser says that the core variable is varied easily by conditions ${ }^{[35]}$ and health quest is no exception. For example, being lied to or rejected in the "bidding" process can extrude people from the system early and delay or prevent medical 
response. Similarly, dwindling resources, a property of illness as omnipotent, limits insurance medical payments, also obstructing the process of care. Once the person transcends hegemony in category 4 by becoming self-sufficient, the presence of an emergency can catapult them back into a need to bid once again for access in a system that extrudes them (a property of category 3 ).

Contested illnesses are destined to revolve through these types of categories until paradigms shift to allow the health systems to acknowledge that our models are limited and to seek answers to questions that require a new understanding of environment and illness. People with chemical and electrical sensitivity seem caught in a time warp where no one is ready to acknowledge, let alone treat them. Consequently they manage disclosure carefully, seek out alternatives, and avoid conventional care unless absolutely forced to access it for emergency needs. McColl and colleagues found high levels of unmet medical needs among people with disabilities, and leveled the relevant comment that "... the system appears to be geared to best serve those who are the easiest to serve." (p. 206). ${ }^{[40]}$

Though a number of international researchers have called for improved services without harm for those with MCS, ${ }^{[41-46]}$ Doiron found that even social service workers who worked with persons with MCS lacked a true understanding of their needs. ${ }^{[4]}$ Given that it is almost impossible to deliver effective medical treatment to persons with MCS in a society where chemical exposure is ubiquitous, we agree with Spencer and Schur when they say,

"The acceptance of MCS would mandate a sig- nificant reconceptualization of risk assessment and regulation of pollutants and other chemicals. If MCS is valid, the precautionary principle ought to be adopted with regulation of potentially harmful chemicals when scientific evidence, although imperfect, is compelling." (p. 26). ${ }^{[48]}$

Until more widespread care is taken regarding toxics, the need for medical care provider training and for accommodation in the medical care setting for those with environmental sensitivities cannot be overstated. Even in the absence of a standard treatment, providers can offer understanding and safe contexts for other medical needs, and can be sounding boards for possible interventions aimed at the sensitivities. In addition, providers can be advocates for patients who need special attention during intrusive procedures, including surgeries, for which no standard MCS accommodations exist. Many with MCS have sensitivities to medications as well, and need careful scrutiny of their reactions to prescriptions.

Limitations of the current study relate to the selection process, as it is not possible to determine if the group that responded differed from the larger group of volunteers. In addition, other researchers might construct different models from the same data. However, we believe that our model captures well the processes of those interviewed, who tried to access a conventional medical system for treatment regarding an unconventional condition.

\section{Conflicts of InTEREST Disclosure}

The authors declare that there is no conflict of interest.

\section{REFERENCES}

[1] Dumit J. Illnesses you have to fight to get: Facts as forces in uncertain, emergent illnesses. Soc Sci Med. 2006; 62: 577-590. PMid: 16085344 http://dx.doi.org/10.1016/j.socscimed.2005.06.018

[2] Ashford NA, Miller CS. Chemical exposures: low levels and high stakes. Second Edition. New York; Van Nostrand Reinhold; 2008.

[3] Gibson PR, Cheavens J, Warren ML. Multiple chemical sensitivity/environmental illness and life disruption. Women Therapy. 1996; 19: 63-79. http://dx.doi.org/10.1300/J015v19n02_06

[4] Multiple chemical sensitivity: a 1999 consensus. Arch Environ Health. 1999; 54(3): 147-149. PMid:10444033 http://dx.doi . org/10.1080/00039899909602251

[5] Gibson PR, Vogel VMR. Sickness related dysfunction in persons with self-reported multiple chemical sensitivity at four levels of severity. J Clin Nurs. 2009; 18: 72-81. PMid:19120734 http: //dx.doi.org/10.1111/j.1365-2702.2008.02439.x

[6] Johansson Å, Brämerson A, Millqvist E, et al. Prevalence and risk factors for self-reported odour intolerance: the Skövde population-based study. Int Arch Occ Env Hea. 2005; 78: 559-564. PMid:16001204 http://dx.doi.org/10.1007/s00420-005-0616-8

[7] Andersson L, Johansson A, Millqvist E, et al. Prevalence and risk factors for chemical sensitivity and sensory hyperreactivity in teenagers. Int J Hyg Envir Heal. 2008; 211(5-6): 690-697. PMid:18403259 http://dx.doi.org/10.1016/j.ijheh.2008.02.002

[8] Berg ND, Linnegarg A, Dirksen A, et al. Prevalence of self-reported symptoms and consequences related to inhalation of airborne chemicals in a Danish population. Int Arch Occ Env Hea. 2008; 81: 881887. PMid:18058120 http://dx.doi.org/10.1007/s00420-0 07-0282-0

[9] Hausteiner C, Bornschein S, Hansen J, et al. Self-reported chemical sensitivity in Germany: a population-based survey. Int J Hyg Envir Heal. 2005; 208: 271-278. PMid:16078641 http://dx.doi.org /10.1016/j.ijheh.2005.03.006

[10] Meggs WJ, Dunn KA, Bloch RM, et al. Prevalence and nature of allergy and chemical sensitivity in a general population. Arch Environ Health. 1996; 51(4): 275-282. PMid:8757407 http://dx.doi.o $\mathrm{rg} / 10.1080 / 00039896.1996 .9936026$ 
[11] Caress S, Steinemann A. A review of a two-phase population study of multiple chemical sensitivities. Environ Health Perspect. 2003; 111: 1490-1497. PMid:12948889 http://dx.doi .org/10.1289 /ehp. 5940

[12] Katerndahl DA, Bell IR, Palmer RF, et al. Chemical intolerance in primary care settings: Prevalence, comorbidity, and outcomes. Ann Fam Med. 2012; 10(4): 357-365. PMid:22778124 http://dx.doi . org/10.1370/afm.1346

[13] Cui X, Lu X, Hiura M, et al. Prevalence and interannual changes in multiple chemical sensitivity in Japanese workers. Environ Health Prev Med. 2014. http://dx.doi.org/10.1007/s12199-014-0 378-6

[14] Dantoft TM, Andersson L, Nordin S, et al. Chemical intolerance. Curr Rheuma Rev. 2015; 11: 167-184. PMid:26088215 http: //dx.doi.org/10.2174/157339711102150702111101

[15] Meggs WJ, Cleveland CH. Rhinolaryngoscopic examination of patients with the Multiple Chemical Sensitivity syndrome. Arch Environ Health. 1993; 48(1): 14-18. PMid:8452394 http://dx.doi.org /10.1080/00039896.1993.9938388

[16] Dantoft TM, Skovbjerg S, Andersson L, et al. Inflammatory mediator profiling of n-butanol exposed upper airways in individuals with Multiple Chemical Sensitivity. PLoS ONE. 2015; 10(11): e0143534. PMid:26599866 http://dx.doi.org/10.1371/journal . pone. 0143534

[17] Mizukoshi A, Kumagai K, Yamamoto N, et al. In-situ Real-Time Monitoring of Volatile Organic Compound Exposure and Heart Rate Variability for Patients with Multiple Chemical Sensitivity. Int J Environ Res Pub Health. 2015; 12: 12446-12465. PMid:26445055 http://dx.doi.org/10.3390/ijerph121012446

[18] Bloch RM, Meggs WJ. Comorbidity patterns of self-reported chemical sensitivity, allergy, and other medical illnesses with anxiety and depression. J Nutr Environ Med. 2007; 16(2): 136-148. http: //dx.doi.org/10.1080/13590840701352823

[19] Gundersen H, Harris A, Bråtveit M, et al. Odor related chronic somatic symptoms are associated with self-reported asthma and hay fever: The Hordaland Health Study. Iran J Allery Asthm. 2015; 14(1): 19-27.

[20] Gibson PR, Lindberg A. Work accommodation for people with multiple chemical sensitivity. Disabil Soc. 2007; 22(7): 717-732. http://dx.doi.org/10.1080/09687590701659576

[21] Vierstra CV, Rumrill PD, Koch LC, et al. Multiple chemical sensitivity and workplace discrimination: The national EEOC ADA research project. 2007; 28: 391-402.

[22] Gibson PR, Sledd LG, McEnroe WH, et al. Isolation and lack of access in multiple chemical sensitivity: A qualitative study. Nurs Health Sci. 2011; 13: 232-237. http://dx.doi.org/10.1111/j $.1442-2018.2011 .00606 . x$

[23] Chircop A, Keddy B. Women living with environmental illness. Health Care Women. 2003; 24: 371-383.

[24] Lipson JG. Multiple chemical sensitivities: Stigma and social experiences. Med Anthropol Q. 2004; 18(2): 200-213. http://dx.doi.o $\mathrm{rg} / 10.1525 / \mathrm{maq} .2004 .18 \cdot 2.200$

[25] Fox M, Kim K. Understanding emerging disabilities. Disabil Soc. 2004; 19(4): 323-3336. http://dx.doi.org/10.1080/0968759 0410001689449

[26] Kristofferzon M, Ternesten-Hasséus E. A study of two generic health-related quality of life questionnaires-Nottingham Health Profile and Short-Form 36 Health Survey-and of coping in patients with sensory hyperreactivity. Heal Qual Life Outcomes. 2013; 11: 182. PMid:24168525 http://dx.doi.org/10.1186/1477-752 5-11-182
[27] Gibson PR, Elms ANM, Ruding LA. Perceived treatment efficacy for conventional and alternative therapies reported by persons with multiple chemical sensitivity. Environ Health Perspect. 2003; 111: 1498-1504. http://dx.doi.org/10.1289/ehp. 5936

[28] Gibson PR, Kovach S, Lupfer A. Unmet healthcare needs for persons with environmental sensitivity. J Multidiscip Health. 2015; 8: 59-66. http://www.dovepress.com/articles.php?artic le_id=20122 PMid:25670904 http://dx.doi .org/10.2147/J MDH. S61723

[29] Johansson O. Electrohypersensitivity: State-of-the-art of a functional impairment. Electromagn Biol Med. 2006; 25: 245258. PMid:17178584 http://dx.doi.org/10.1080/153683706 01044150

[30] Clarke J, James S. The Radicalized self: the impact on the self of the contested nature of the diagnosis of chronic fatigue syndrome. Soc Sci Med. 2003; 57: 1387-1395. http://dx.doi.org/10.1016/S 0277-9536 (02) 00515-4

[31] Crooks VA. Exploring the altered daily geographies and lifeworlds of women living with fibromyalgia syndrome: a mixed-method approach. Soc Sci Med. 2007; 64: 577-588. PMid:17079063 http: //dx.doi.org/10.1016/j. socscimed.2006.09.025

[32] Gibson PR, Placek E, Lane J, et al. Disability induced identity changes in persons with multiple chemical sensitivity. Qual Health Res. 2005; 15(4): 502-524. PMid:15761095 http://dx.doi.org $/ 10.1177 / 1049732304271960$

[33] Swoboda DA. The social construction of contested illness legitimacy: A grounded theory analysis. Qual Res Psych. 2006; 3: 233-251. http://dx.doi.org/10.1191/1478088706qrp061oa

[34] Glaser BG. Doing grounded theory: Issues and Discussions. Mill Valley, CA: Sociology Press; 1998.

[35] Glaser BG. Theoretical sensitivity: advances in the methodology of grounded theory. Mill Valley CA: Sociology Press; 1978.

[36] Introductory Guide to Critical Theory. Available from: https://www.cla.purdue.edu/english/theory/marx ism/terms/hegemony.html

[37] Gibson PR, Lindberg A. Physicians' perceptions and practices regarding patient reports of chemical sensitivity. ISRN Nursing. 2011. 1-5. PMid:22007328 http://dx.doi .org/10.5402/2011/838930

[38] Joachim G, Acorn S. Stigma of visible and invisible chronic conditions. J Adv Nurs. 2000; 32(1): 243-248. PMid:10886457 http: //dx.doi.org/10.1046/j.1365-2648.2000.01466.x

[39] Jussila AL. Stabililising of life: Families living with cancer. In B.G. Glaser, \& J.A. Holton (Eds.). The Grounded Theory Reader. Mill Valley, CA; Sociology Press; 2007.

[40] McColl MA, Jarzynowskab A, Shortt SED. Unmet health care needs of people with disabilities: population level evidence. Disabil Soc. 2010; 25(2): 205-218. http://dx.doi.org/10.1080/0968759 0903537406

[41] Cooper C. Multiple chemical sensitivity in the clinical setting: although the cause and diagnosis of this condition remain controversial, the patient's concerns should be heeded. Amer J Nurs. 2007; 107(3): 40-47. http://dx.doi.org/10.1097/0000044 6-200703000-00020

[42] Glinton G. Multiple-chemical sensitivity. Med Surg Nursing. 2005; 14(6): 365-370. PMid:16447825

[43] Imai N, Imai Y, Kido Y. Psychosocial factors that aggravate the symptoms of sick house syndrome in Japan. Nurs Health Sci. 2008; 10: 101-109. PMid:18466382 http://dx.doi.org/10.1111/j. 144 2-2018.2008.00389.x

[44] Ishikawa S, Miyata M. Chemical sensitivity and its clinical characteristics in Japan. Asian Med J. 2000; 43(1): 7-15. 
[45] Larsson C, Martensson L. Experiences of problems in individuals with hypersensitivy to odours and chemicals. J Clin Nurs. 2009; 18: 737-744. PMid:19239540 http://dx.doi.org/10.1111/j.136 5-2702.2008.02599.x

[46] Lavergne MR, Cole DC, Kerr K, et al. Functional impairment in chronic fatigue syndrome, fibromyalgia, and multiple chemical sensi- tivity. Can Fam Physician. 2010; 56: 57-65.

[47] Doiron N. People with environmental sensitivities: Life, identity, and services. Doctoral Thesis in Social Work. University of Toronto. 2007.

[48] Spencer TR, Schur PM. The challenge of multiple chemical sensitivity. J Environ Heal. 2008; 70(10): 24-27. PMid:18561566 